\title{
Cost-effectiveness of nivolumab in patients with advanced renal cell carcinoma treated in the United States
}

\author{
Charles McCrea ${ }^{1^{*}}$ (D), Sukhvinder Johal', Shuo Yang ${ }^{2}$ and Justin Doan ${ }^{2}$
}

\begin{abstract}
Background: We evaluated the cost-effectiveness of nivolumab versus everolimus in patients with advanced renal cell carcinoma (RCC) from a US payer perspective.

Methods: A partitioned survival model consisting of three health states, progression-free survival (PFS), progressive disease, and death, was developed to evaluate the cost-effectiveness of intravenous nivolumab versus oral everolimus over a lifetime. The proportion of patients in each state was calculated based on parametric distributions fitted to PFS and overall survival (OS) data from CheckMate $025(\mathrm{~N}=821)$, a large randomized phase 3 trial of nivolumab versus everolimus for advanced RCC. Health state utility data were derived from CheckMate 025 EQ-5D data. Scenario analyses and deterministic and probabilistic sensitivity analyses assessed the impact of uncertainty in model inputs on outcomes.

Results: Over a 25-year lifetime horizon, treatment with nivolumab resulted in a gain of 0.64 quality-adjusted lifeyears (QALYS) versus everolimus. Nivolumab had greater total costs versus everolimus (\$US197,089 vs. \$US163,902), mainly due to higher acquisition costs. The incremental cost-utility ratio (ICUR), a measure of incremental costs divided by incremental QALYS, was $\$$ US51,714 per QALY gained for nivolumab versus everolimus, and an incremental cost-effectiveness ratio was $\$$ US44,576 per life-year gained for nivolumab versus everolimus. In sensitivity analyses, average body weight had the greatest impact on the ICUR for nivolumab versus everolimus (base case $\$$ US51,714; range \$US8863-\$US94,566). At a \$US150,000 willingness-to-pay (WTP) threshold, nivolumab had a 91.7\% probability of being cost-effective versus everolimus.
\end{abstract}

Conclusions: In the United States, at a WTP threshold of $\$$ US150,000 per QALY, nivolumab was found to be costeffective. Key drivers of cost-effectiveness were survival inputs for OS and the average weight of patients; the latter directly affects nivolumab drug acquisition cost.

Keywords: Cost-effectiveness, Everolimus, Nivolumab, Renal cell carcinoma, Survival model

\section{Background}

In the United States, kidney cancer is the sixth most common cancer in men and tenth most common cancer in women [1]. In 2017, there were $~ 64,000$ new cases and $\sim 14,500$ deaths as a result of kidney cancer [1]. Renal cell carcinoma (RCC) accounts for $\sim 80 \%$ of all kidney cancers [2]. In addition to low survival rates for advanced

\footnotetext{
*Correspondence: Charles.McCrea@parexel.com

1 Health Economic Modelling Unit, PAREXEL Access Consulting, Evergreen Building North, 160 Euston Road, London NW1 2DX, UK

Full list of author information is available at the end of the article
}

disease [3], there is also a substantial economic burden due to kidney cancer in the United States [4].

Nivolumab is a first-in-class programmed death-1 immune checkpoint inhibitor approved for use in patients with advanced RCC who have received prior antiangiogenic therapy. Approval was based on results from CheckMate 025, a randomized phase 3 trial of nivolumab $3 \mathrm{mg} / \mathrm{kg}$ every 2 weeks $(\mathrm{N}=410)$ versus everolimus $10 \mathrm{mg}$ once daily $(\mathrm{N}=411)$ in patients with previously treated advanced RCC [5]. The study met its primary endpoint of overall survival (OS), achieving 
a median OS of 25.0 months with nivolumab versus 19.6 months with everolimus [5]. Nivolumab was associated with a significantly improved adverse event (AE) profile. Grade 3-4 treatment-related AEs were 19\% for nivolumab versus $37 \%$ for everolimus [5]. Longer-term survival data are available for a phase 1 (CA209-003) study of nivolumab, which demonstrated a median OS of 22.4 months and a 5 -year OS rate of 34\% (minimum follow-up, 50.5 months) in patients with advanced RCC [6].

With the increased use of nivolumab as a standard of care in second-line RCC, there is a need to inform US payers on the value of nivolumab. Although cost-effectiveness analyses have been conducted for nivolumab in melanoma and non-small cell lung cancer in a number of countries [7-11], few analyses have been reported for RCC $[12,13]$.

The objectives of the current study were to evaluate the cost-effectiveness of nivolumab compared with everolimus in patients with advanced RCC from a US payer perspective. To this end, a decision analytical model was developed and validated to estimate the lifetime costs and outcomes of treatments for this patient population.

\section{Patients and methods}

\section{Health state model structure}

Consistent with the approaches adopted in previous economic evaluations and technology appraisals for RCC treatment that were submitted to the Scottish Medicines Consortium and the National Institute for Health and Care Excellence, a 3-state partitioned survival model was developed to evaluate the incremental cost-utility ratio (ICUR) and incremental cost-effectiveness ratio (ICER) of nivolumab versus everolimus. The model comprised 3 key health states representing the primary stages of disease in advanced or metastatic RCC: progression-free $(\mathrm{PF})$, progressive disease (PD), and death. Each state represents a point in the disease pathway at which healthrelated quality of life (HRQoL) is expected to vary.

The partitioned survival model estimated occupancy of health states using an area-under-the-curve approach from OS and progression-free survival (PFS) parametric curves derived from CheckMate 025. Patient-level data from CheckMate 025 (minimum follow-up, 26 months) were used in the development and validation of the economic model outputs. CheckMate 025 was a large, randomized phase 3 study of nivolumab versus everolimus for previously treated advanced RCC. The time horizon of the analysis was defined as a lifetime ( 25 years), and a 4-week cycle length (a fixed unit of time by which the model moves forward in time) was selected to accommodate the dosing regimens for nivolumab and everolimus.

\section{Survival estimates}

CheckMate 025 provided data up to a limited time period (minimum follow-up, 26 months; median follow-up, 33.6 months). In order to adopt a lifetime perspective, extrapolation beyond the trial period was required to estimate disease progression and OS over a lifetime. A number of models exist for extrapolating survival. Each model has a different functional form and makes different underlying assumptions on the long-term behavior of survival data, resulting in different survival estimates (and hence ICURs/ICERs). The National Institute for Health and Care Excellence Decision Support Unit has issued guidance on appropriate selection of the extrapolation method, and we followed this selection process to identify appropriate models for nivolumab and everolimus [14]. The fitted curves were assessed in terms of visual inspection of survival curve fit to clinical trial KaplanMeier data, inspection of log-cumulative hazard plots (to assess the behavior of the hazards over time), statistical model fit via measures such as Akaike's information criterion (AIC)/Bayesian information criterion (BIC), and long-term extrapolation estimates versus longer-term data from the phase 1 CA209-003 study (60-month OS time point) of nivolumab for previously treated advanced RCC [6]. Based on the goodness of fit, OS was modeled using the dependent 1-knot spline normal survival function for nivolumab and everolimus (Fig. 1, top panel). PFS was modeled using the dependent 2-knot spline hazard for nivolumab and everolimus, with an adjustment on the shape parameter at gamma 1 and 2 for nivolumab (Fig. 1, bottom panel). Parametric spline models are "structurally flexible" extensions of the standard parametric distributions such as the Weibull, log-normal, and log-logistic functions. They are similar to piecewise modeling as they are flexible mathematical functions defined by piecewise polynomials joined at time points along the curve known as knots. They are particularly useful in cost-utility modeling as they are more flexible models that can better fit the estimated Kaplan-Meier data from clinical trials, particularly when the Kaplan-Meier curves are "unique" and difficult to fit with standard distributions, or when several clinical processes influence the shape of the curve. In the case of nivolumab, spline-based models provided a better fit to the observed data compared with other, more standard parametric models. Other models were tested in a sensitivity analysis.

\section{Health-related quality of life (utility) estimates}

The EuroQol-5 Dimensions (EQ-5D) instrument was used in the CheckMate 025 study to collect HRQoL data by health state. Health state utilities for PF for nivolumab 

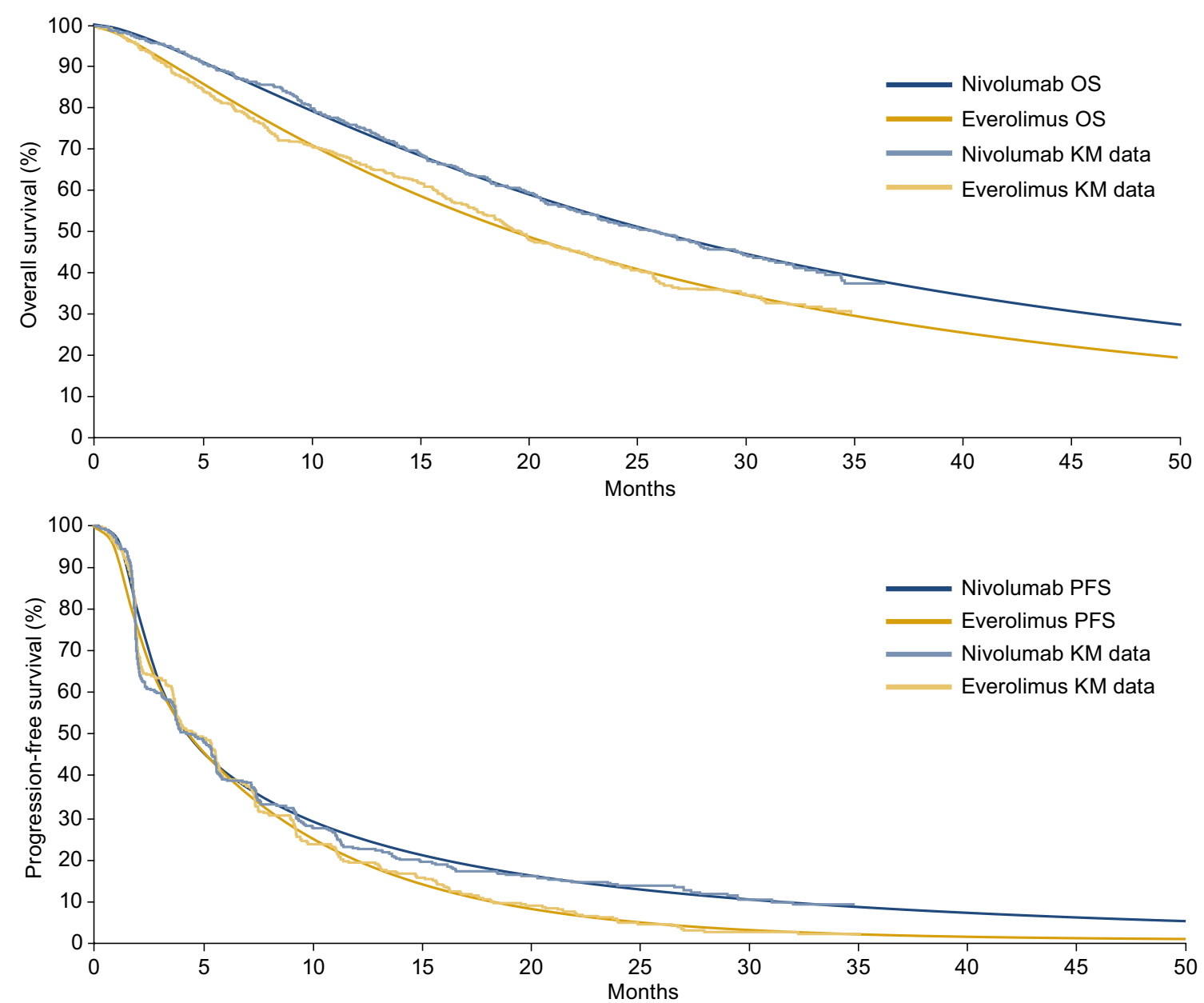

Fig. 1 OS (top panel) and PFS (bottom panel) Kaplan-Meier curves with best-fitting parametric curves. KM, Kaplan-Meier; OS, overall survival; PFS, progression-free survival

and everolimus were weighted according to the objective response rate estimated for each treatment from CheckMate 025. The odds ratio for treatment response was 5.93 for nivolumab versus everolimus. It was assumed in the economic model that all patients remaining in the PF health state beyond 22 months were considered to have the health state utility of responders. The utility values assigned to each health state were as follows: PF (complete response/partial response), 0.895; PF (stable disease), 0.846; and PD, 0.817. Additional one-off utility decrements were ascribed to patients who experienced AEs.

\section{Cost estimates}

Cost estimates were derived based on the US healthcare system. Only direct medical costs were included in the analysis. These included the cost of drug acquisition, drug administration, monitoring (routine scans), disease management, end-of-life care, and the management of
AEs. Patients were treated until progression, although this assumption was tested in a sensitivity analysis. The drug acquisition cost for $10 \mathrm{mg} / \mathrm{mL}$ nivolumab was \$US2545.15 per vial for $100 \mathrm{mg}$, and \$US13,233.17 for $10 \mathrm{mg}$ of everolimus in 28 tablets [15]. The drug acquisition cost per administration of nivolumab without wastage was \$US5451.71. The incidence rates of AEs in the model were derived from CheckMate 025 and included any drug-related grade 3 or higher events with a $>5 \%$ incidence in either the nivolumab or everolimus arm. AEs included in the model were hypertriglyceridemia and anemia; the unit cost per event was \$US73.06 and \$US3237.89, respectively [16, 17]. US-specific inputs related to costs (cost year, 2017) were disease management costs, drug acquisition costs, and unit administration costs (\$US139.61 for nivolumab [18]; zero for everolimus because it is orally administered) and routine monitoring costs with the assumption of 1 oncologist visit every 4 weeks (\$US79.67) (Additional file 1: Tables 
S1, S2) [19]. In CheckMate 025, 55\% of patients treated with nivolumab and $63 \%$ of patients treated with everolimus received subsequent systemic therapy [5]. A subsequent treatment cost was applied as a one-off cost to patients entering the PD health state and was calculated based on therapies received by $>10$ patients in CheckMate 025 and an average of 3.65 months' duration of treatment (Additional file 1: Table S3) [20].

\section{Scenario and sensitivity analyses}

Scenario, deterministic sensitivity, and probabilistic sensitivity analyses were conducted to assess the impact of uncertainty in model inputs on the outcomes. Two scenario analyses were carried out. First, a commonly used standard parametric distribution, a Weibull model, was independently fitted to everolimus and nivolumab OS data. Second, because CheckMate 025 had a significant number of patients treated post progression, the time to treatment discontinuation (TTD) data derived from CheckMate 025 were used in lieu of PFS data in the nivolumab arm to account for differences in PFS and observed TTD which may have resulted due to issues of pseudoprogression in the nivolumab cohort [21]. Oneway deterministic sensitivity analyses were conducted with means and plausible ranges for each parameter

Table 1 Inputs for deterministic sensitivity analysis

\begin{tabular}{ll}
\hline Parameter & Mean (range) \\
\hline Discount rate_costs, \% & $3(0-6)$ \\
Discount rate_outcomes, \% & $3(0-6)$ \\
Average body weight, kg & $71.4(57.1-85.7)$ \\
Cost_PF state, \$US & $65.67(52.53-78.80)$ \\
Cost_PD state, \$US & $91.61(73.29-109.93)$ \\
Terminal cost, \$US & $10,713.01(8570.41-12,855.61)$ \\
Administration cost, \$US & \\
$\quad$ Nivolumab & $139.61(111.69-167.53)$ \\
Everolimus & Not applicable \\
Monitoring cost, \$US & \\
$\quad$ Nivolumab & $79.67(63.74-95.60)$ \\
Everolimus & $79.67(63.74-95.60)$ \\
Utility weight, PF, response & \\
$\quad$ Nivolumab & $0.895(0.889-0.901)$ \\
Everolimus & $0.895(0.889-0.901)$ \\
Utility weight, PF, no response & \\
Nivolumab & $0.846(0.840-0.852)$ \\
Everolimus & $0.846(0.840-0.852)$ \\
Utility weight, PD & \\
Nivolumab & $0.817(0.811-0.823)$ \\
Everolimus & $0.817(0.811-0.823)$ \\
\hline PD progressivedisease &
\end{tabular}

$\mathrm{PD}$, progressive disease health state; $\mathrm{PF}$, progression-free health state

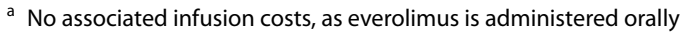

(Table 1). The following parameters were used: discount rate, body weight, costs, utilities, and clinical data.

Probabilistic sensitivity analysis was used to assess the variation in the model results from the uncertainty around each parameter in the model. Model parameters were sampled from parametric distributions to generate 1000 estimates of the costs and effects in each treatment arm. A gamma distribution was adopted for all costs and resource utilization parameters and a beta distribution was used for the utilities and all probabilities [22]. Drug acquisition costs were exempt from the probabilistic analysis. For the parametric survival curves for PFS and OS, a multivariate normal distribution with correlation between curve parameters was used [22].

\section{Results}

Base-case results

Over a 25-year horizon, treatment with nivolumab resulted in a quality-adjusted life-year (QALY) gain of 0.64 versus everolimus (Table 2 ).

Patients treated with nivolumab incurred higher costs, mainly associated with treatment acquisition, in part due to the increased time spent in the PF health state (Table 2). The ICUR of \$US51,714 per QALY gained

Table 2 Base-case results for the United States

\begin{tabular}{|c|c|c|c|}
\hline & Nivolumab & Everolimus & $\begin{array}{l}\text { Incremental } \\
\text { results }\end{array}$ \\
\hline Total costs, \$US & 197,089 & 163,902 & 33,186 \\
\hline \multicolumn{4}{|l|}{ Health states, \$US } \\
\hline PF & 833 & 539 & \\
\hline PD & 12,596 & 12,382 & \\
\hline \multicolumn{4}{|l|}{ Initial treatment, \$US } \\
\hline Acquisition & 138,429 & 108,859 & \\
\hline Administration & 3545 & 0 & \\
\hline Monitoring & 1011 & 655 & \\
\hline \multicolumn{4}{|l|}{ Subsequent treatment, \$US } \\
\hline Acquisition & 40,272 & 40,817 & \\
\hline Administration & 92 & 127 & \\
\hline Monitoring & 254 & 265 & \\
\hline Adverse events, \$US & 56 & 257 & \\
\hline Total QALYs (discounted) & 2.79 & 2.15 & 0.64 \\
\hline PF & 0.84 & 0.54 & \\
\hline PD & 1.95 & 1.62 & \\
\hline AEs & -0.001 & -0.006 & \\
\hline Total LYG (discounted) & 3.36 & 2.61 & 0.74 \\
\hline $\begin{array}{l}\text { Incremental cost per QALY } \\
\text { gained, \$US }\end{array}$ & & & 51,714 \\
\hline $\begin{array}{l}\text { Incremental cost per LYG, } \\
\text { \$US }\end{array}$ & & & 44,576 \\
\hline
\end{tabular}

$A E$, adverse event; $L Y G$, life-year gained, $P D$; progressive disease health state; $P F$, progression-free health state; QALY, quality-adjusted life-year 
versus everolimus, and an ICER of \$US44,576 per lifeyears gained (LYG) versus everolimus, were estimated (Table 2). AE treatment costs were lower with nivolumab versus everolimus (Table 2).

\section{Scenario analyses}

An investigation into the effect of parametric curve selection on costs, HRQoL, and survival was conducted using Weibull curves fitted separately to nivolumab and everolimus data. The incremental cost per QALY for nivolumab versus everolimus was $\$$ US80,439, and the incremental cost per LYG was \$US71,697. Incremental costs for nivolumab versus everolimus were \$US31,457 and incremental QALYs were 0.39 (Table 3). In a second scenario analysis, a TTD curve was used as proxy for PFS in the nivolumab arm only. When both costs and QALYs were calculated using TTD instead of PFS for nivolumab, the incremental cost per QALY for nivolumab versus everolimus was $\$$ US99,574 and the incremental cost per LYG was \$US87,391. Incremental costs were \$US65,062 and incremental QALYs were 0.65 (Table 3).

\section{Deterministic and probabilistic sensitivity analyses}

The average body weight had the greatest impact on the ICUR for nivolumab versus everolimus (base case \$US51,714; range \$US8863-\$US94,566; Fig. 2). The ICUR was also sensitive to the discount rate for costs and outcomes (range \$US42,771-\$US63,179 and \$US43,288$\$$ US60,077, respectively). The ICUR did not change substantially across the other parameters tested.

Probabilistic sensitivity analysis was conducted to assess the variation in the model results from the uncertainty around each parameter tested. The cost-effectiveness scatterplot for nivolumab versus everolimus is shown in Fig. 3.

At a willingness-to-pay threshold of $\$ \mathrm{US} 150,000$, nivolumab had a $91.7 \%$ probability of being cost-effective compared with everolimus alone in the base-case setting. At a willingness-to-pay threshold of $\$ U S 200,000$, nivolumab had a $96.7 \%$ probability of being cost-effective compared with everolimus (Fig. 4).

The best-fitting parametric survival models were validated against observed data from CheckMate 025 (minimum follow-up, 26 months) and 60-month survival rates derived from the phase 1 CA209-003 study [5, 6]. Parametric survival models match the Kaplan-Meier data from the clinical trials closely (Table 4).

Based on the observed 60-month OS rate with nivolumab in CA209-003 (34.3\%) [6], the 60-month OS rate estimated using the base-case scenario (dependent 1-knot spline normal) may underestimate survival (22.4\%) (Table 4). Scenarios using the dependent loglogistic (23.8\%) and independent Weibull distributions may also underestimate survival (15.6 and $11.0 \%$ for nivolumab and everolimus, respectively) compared with the observed 60-month OS rate in CA209-003 (Table 4).

\section{Discussion}

The results of the economic evaluation described here indicate that at a cost of \$US2545.15 per 100-mg vial, nivolumab has an ICUR of \$US51,714 per QALY gained over everolimus and an ICER of \$US44,576 per LYG over everolimus. At a willingness-to-pay threshold of $\$$ US150,000, nivolumab had a $91.7 \%$ probability of being cost-effective compared with everolimus. The key drivers of cost-effectiveness were the survival inputs for OS and the average weight of patients, the latter having a direct effect on the drug acquisition cost of nivolumab. Validation with long-term RCC survival data in the real-world setting is limited due to lack of data beyond 5 years. Although the dependent 1-knot spline normal is the bestfitting curve for OS observed in CheckMate 025 based on AIC and BIC criteria, the long-term projections indicate that the dependent log-logistic curves may provide a better fit, but are still a conservative assumption compared with CA209-003 5-year data. However, it should be noted that although CA209-003 provides long-term survival estimates, the trial population included previously treated patients who received a range of doses of nivolumab [6], which may explain some of the differences in long-term survival versus CheckMate 025 projections.

For this economic evaluation, the wholesale acquisition cost of nivolumab was used. However, the actual price of nivolumab in the United States may be lower, according to price negotiations with pharmacies. Therefore, the results presented may be considered conservative.

The cost-effectiveness of nivolumab was explored in Sweden using a 3-state partitioned survival model [12]. Nivolumab was found to be cost-effective at established thresholds when accounting for treatment costs that continued through progression [12]. In contrast, Wan et al. concluded that nivolumab was not cost-effective from US and Chinese healthcare system perspectives [13]. Results from the current analysis differed, likely because there were some key differences between approaches, with the current analysis utilizing individual patient-level data and employing a robust methodology to select appropriate distributions to model OS that underwent further validation using long-term CA209-003 study data. Wan et al. used a Weibull distribution to model OS independently for the nivolumab and everolimus arms, with the rationale that it is widely used in cancer survival analyses. There did not appear to be any consideration of goodness of fit against the trial data compared with other parametric models or any attempt to validate longer-term OS predictions. As part of a scenario analysis, we used 


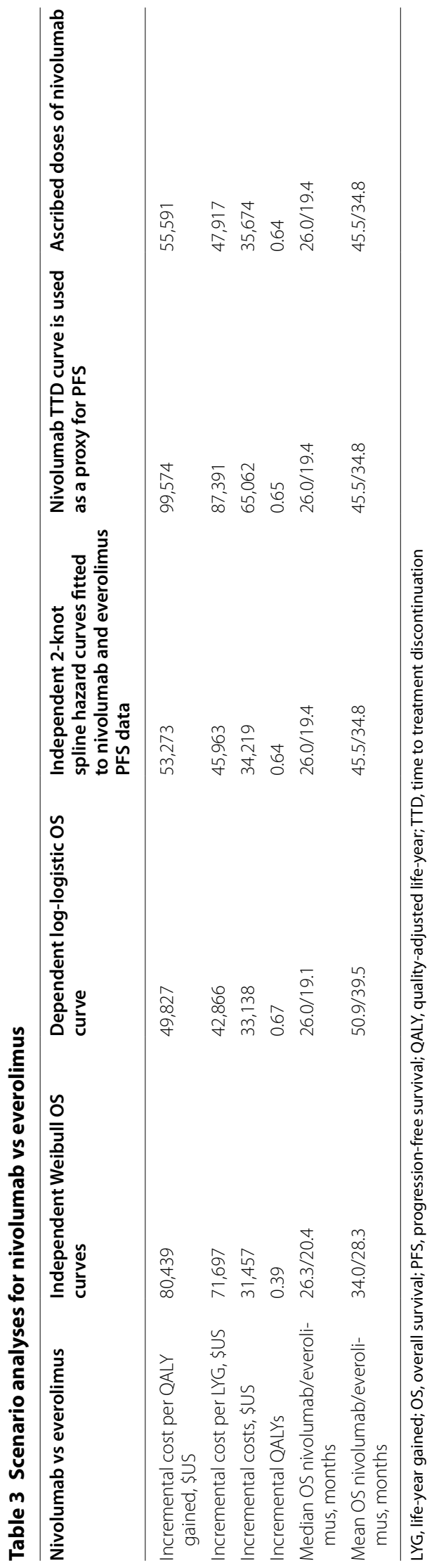




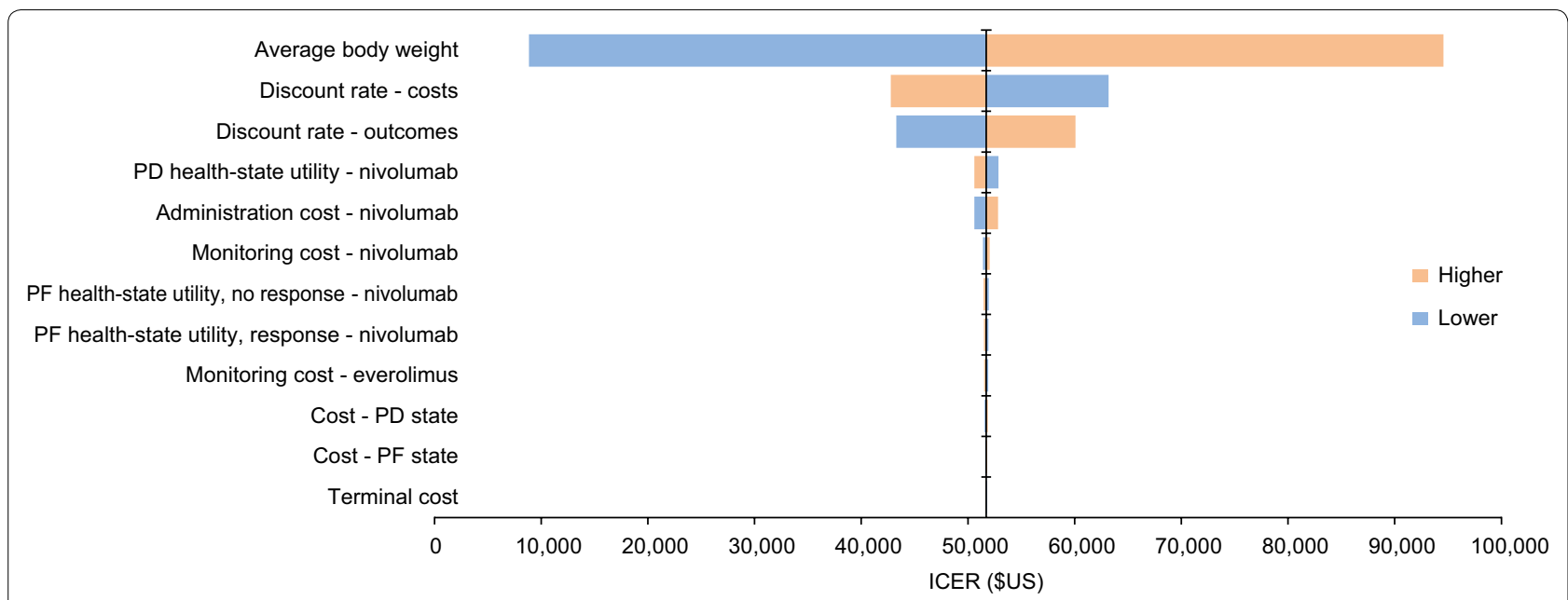

Fig. 2 Deterministic sensitivity analysis. Orange bars represent the upper bound of each parameter varied. Blue bars represent the lower bound of each parameter varied. ICER, incremental cost-effectiveness ratio; PD, progressive disease; PF, progression-free

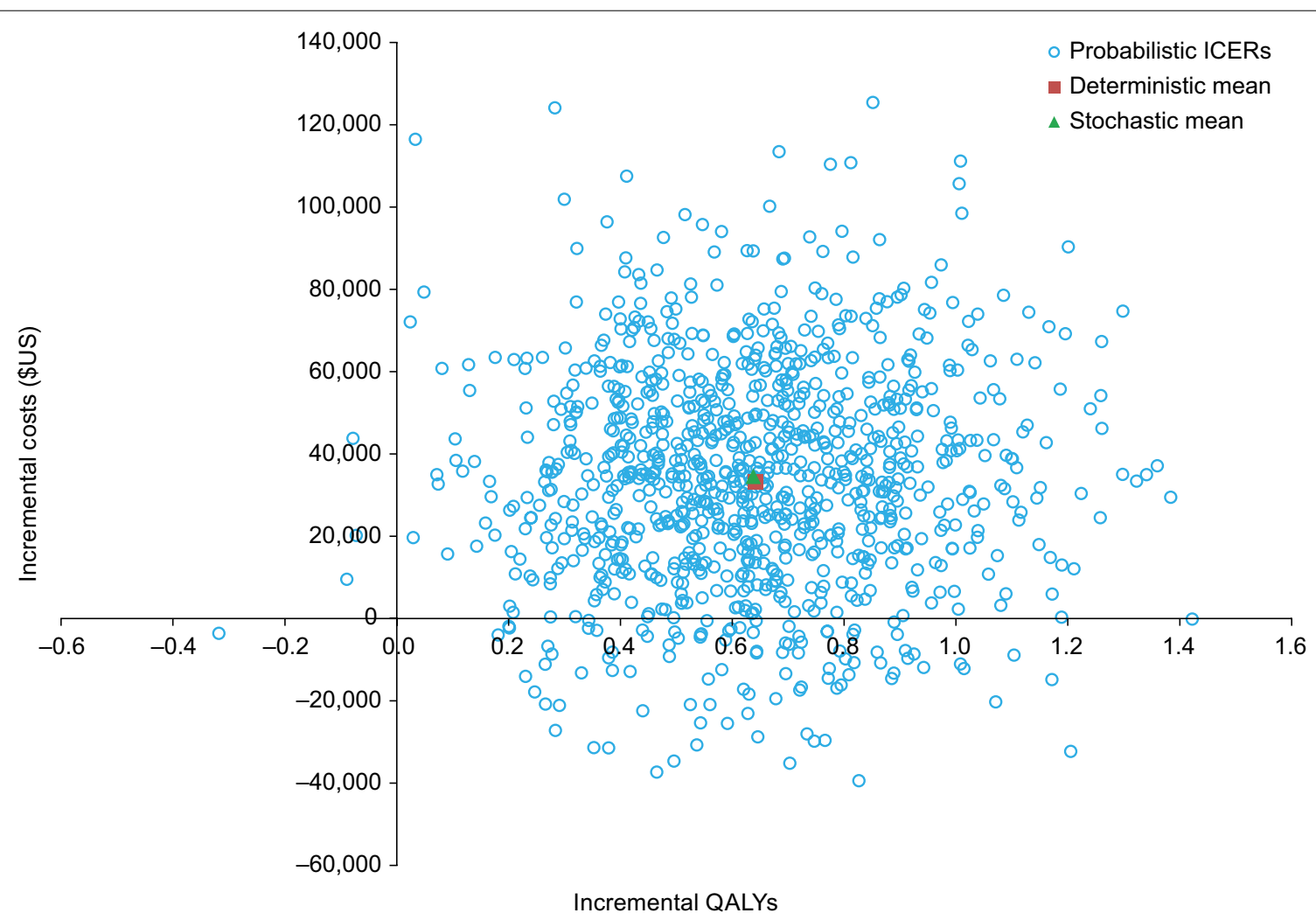

Fig. 3 Cost-effectiveness plane (nivolumab vs. everolimus). ICER, incremental cost-effectiveness ratio; QALY, quality-adjusted life-year

independent Weibull distributions for OS. The Weibull distribution resulted in a lower mean OS for nivolumab and everolimus and did not reflect the long-term survival benefit of nivolumab observed in CA209-003, providing much lower 60-month survival estimates versus the dependent 1-knot spline normal survival function used in the base-case analysis. Wan et al. also used a less mature dataset from CheckMate 025, which may have resulted in a higher degree of uncertainty in longer-term OS extrapolations. 


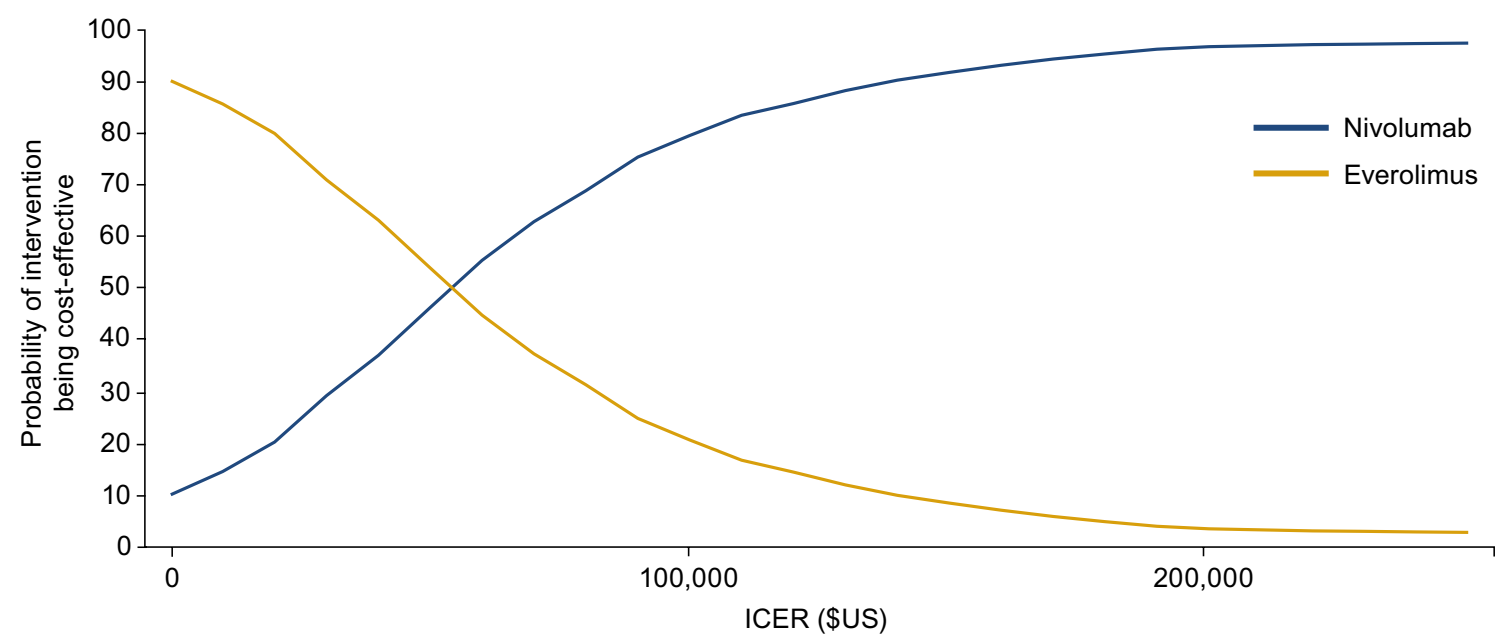

Fig. 4 Pair-wise cost-effectiveness acceptability curve (nivolumab vs. everolimus). ICER, incremental cost-effectiveness ratio

There are several limitations to our analysis that deserve consideration. The economic model utilizes PFS as a proxy for treatment duration. However, in the nivolumab arm, there were differences in the PFS and TTD curves, indicating that a proportion of patients were treated post progression. Some of those patients may have had pseudoprogression, an observed phenomenon caused by the unconventional immune-related response that gives the initial appearance of progression [23]. Thus, the costs and benefits of nivolumab treatment may be underestimated in the base-case analysis. The ICUR increased substantially when the TTD curve was used to calculate drug acquisition, drug administration, and monitoring costs for nivolumab; however, it is important to consider that the quality-of-life benefits associated with increased treatment duration on nivolumab were not captured in this scenario. In addition, only AEs with $>5 \%$ incidence at grade 3 or higher severity were included. This could underestimate the impact of AEs in the model because nivolumab has a more favorable tolerability profile than everolimus. However, it should be noted that AEs contributed to $<1 \%$ of the incremental costs of nivolumab versus everolimus. Finally, long-term extrapolation of OS curves from short-term clinical trials is always subject to uncertainty, and hence should be validated against long-term data from other sources. However, long-term validation proved difficult due to a lack of real-world evidence for advanced RCC. Where data were available, the model was validated against long-term survival data and different parametric distributions for OS were tested in scenario analyses.

The standard dose of nivolumab for RCC was recently modified from $3 \mathrm{mg} / \mathrm{kg}$ every 2 weeks to $240 \mathrm{mg}$ every 2 weeks [24] based on population pharmacokinetics and dose/exposure-response analyses. A dose and schedule of $480 \mathrm{mg}$ every 4 weeks is currently being explored, which may improve the cost-effectiveness of nivolumab in this patient population because fewer office visits would be needed for drug administration.

The results of the economic evaluation need to be considered in the context of the high unmet need/poor survival within previously treated advanced RCC [2]. The 5 -year survival for metastatic RCC is $11.7 \%$ [3]. There is also a substantial cost of illness for metastatic RCC, with patients experiencing high out-of-pocket costs [25-27]. Effective strategies to reduce the prevalence and progression of RCC have the potential to considerably reduce the economic burden. Studies on treatment sequence and schedule that may help to maximize benefit and optimize cost-effectiveness should be explored. Clinical data of nivolumab in previously treated patients with advanced RCC presents a compelling case that nivolumab represents a "step-change" in disease management with improved OS, favorable tolerability, and improved quality of life compared with everolimus [5, 28].

At this juncture, physicians and payers alike are making second-line treatment decisions for patients with RCC with nivolumab and cabozantinib in mind. This analysis may be extended to an indirect comparison of cost-effectiveness of these two agents.

\section{Conclusions}

In addition to the known survival benefit, improved HRQoL, and favorable tolerability profile of nivolumab compared with everolimus, we also found nivolumab to be cost-effective for patients with advanced RCC at a \$US150,000 per QALY threshold in the United States, even when taking into account the higher costs of initial 


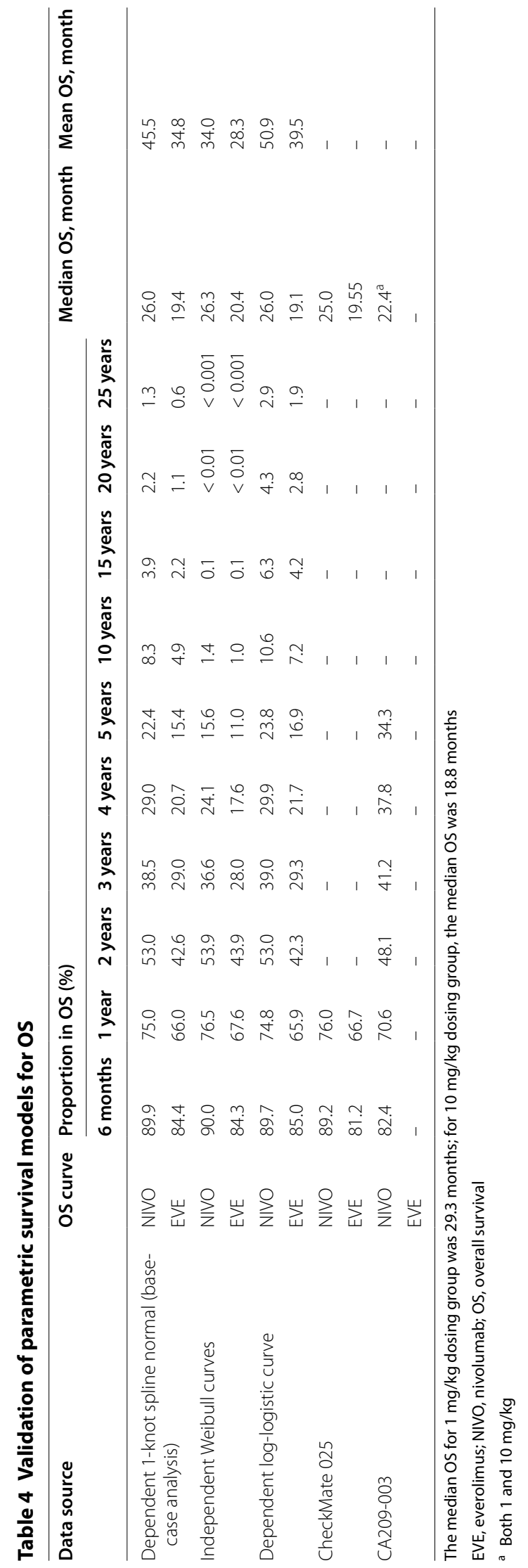


treatment acquisition, administration, and monitoring. The model structure developed here may serve as a template for assessing cost-effectiveness of nivolumab in other countries and for comparison with other relevant treatments for second-line RCC.

\section{Additional file}

Additional file 1. Additional tables.

\section{Abbreviations}

AE: adverse event; AIC: Akaike's information criterion; BIC: Bayesian information criterion; EQ-5D: EuroQol-5 Dimensions; HRQoL: health-related quality of life; ICER: incremental cost-effectiveness ratio; ICUR: incremental cost-utility ratio; LYG: life-years gained; OS: overall survival; PD: progressive disease; PF: progression-free; PFS: progression-free survival; QALY: quality-adjusted lifeyear; RCC: renal cell carcinoma; TTD: time to treatment discontinuation.

\section{Authors' contributions}

Conceptualization; formulation of overarching research goals and aims: JD and SY. Methodology; development or design of methodology, creation of models: CMC, SJ, SY and JD. Software; programming, software development; designing computer programs; implementation of the computer code and supporting algorithms; testing of existing code components: CMC and SJ. Validation; verification, whether as a part of the activity or separate, of the overall replication/reproducibility of results/experiments and other research outputs: CMC and SJ. Formal analysis; application of statistical, mathematical, computational, or other formal techniques to analyze or synthesize study data: CMC and SJ. Investigation; research and investigation process, specifically performing the experiments, or data/evidence collection: CMC and SJ. Resources; provision of study materials, reagents, materials, patients, laboratory samples, animals, instrumentation, computing resources, or other analysis tools: CMC, SJ, SY and JD. Data curation; management activities to annotate (produce metadata), scrub data and maintain research data (including software code, where it is necessary for interpreting the data itself) for initial use and later re-use: CMC and SJ. Writing, original draft; preparation, creation and/or presentation of the published work, specifically writing the initial draft (including substantive translation): CMC and SJ. Writing, review and editing; preparation, creation and/or presentation of the published work by those from the original research group, specifically critical review, commentary or revision-including pre- or post-publication stages: CMC, SJ, SY and JD. Visualization; preparation, creation, and/or presentation of the published work, specifically visualization/ data presentation: CMC, SJ, SY and JD. Supervision; oversight and leadership responsibility for the research activity planning and execution, including mentorship external to the core team: JD and SY. Project administration; management and coordination responsibility for the research activity planning and execution: JD and SY. Funding acquisition; acquisition of the financial support for the project leading to this publication: JD and SY. All authors contributed to the manuscript. All authors read and approved the final manuscript.

\section{Author details}

${ }^{1}$ Health Economic Modelling Unit, PAREXEL Access Consulting, Evergreen Building North, 160 Euston Road, London NW1 2DX, UK. ${ }^{2}$ Bristol-Myers Squibb, Princeton, NJ, USA.

\section{Acknowledgements}

Medical writing assistance was provided by Jennifer Granit, Ph.D., of PPSI (a PAREXEL company), funded by Bristol-Myers Squibb.

\section{Competing interests}

Sukhvinder Johal and Charles McCrea are employees of PAREXEL Access Consulting. PAREXEL Access Consulting received consultancy fees from BristolMyers Squibb to develop the economic model and conduct analyses. Justin Doan and Shuo Yang are employees of and hold stock options in Bristol-Myers Squibb.

\section{Availability of data and materials}

The model underlying this paper was developed in Excel. The model file is not publicly available as it contains additional calculations and results that are not yet in the public domain, but which will feature in future publications that build on this work. The full details of the model structure and derivation of inputs are presented in the paper and additional file.

\section{Consent for publication}

Not applicable.

\section{Ethics approval and consent to participate}

The CheckMate 025 study was approved by the institutional review board or an independent ethics committee at each center. Ethics approval was not required for the portion of the study described herein. Bristol-Myers Squibb acts as the overall guarantor for this work.

\section{Funding}

This work was sponsored by Bristol-Myers Squibb. The funders contributed to the design and conduct of the study; collection, management, analysis, and interpretation of the data; and preparation, review, and approval of the manuscript in collaboration with the authors of this report.

\section{Publisher's Note}

Springer Nature remains neutral with regard to jurisdictional claims in published maps and institutional affiliations.

Received: 22 November 2017 Accepted: 2 February 2018

Published online: 09 February 2018

\section{References}

1. Siegel RL, Miller KD, Jemal A. Cancer statistics, 2017. CA Cancer J Clin. 2017:67:7-30

2. Escudier B, Porta C, Schmidinger M, et al. Renal cell carcinoma: ESMO clinical practice guidelines for diagnosis, treatment and follow-up. Ann Oncol. 2016;27:v58-68.

3. Surveillance, Epidemiology, and End Results Program. SEER cancer stat facts: kidney and renal pelvis cancer. https://seer.cancer.gov/statfacts/ html/kidrp.html. Accessed 1 May 2017.

4. Shih YC, Chien $C R$, Xu Y, et al. Economic burden of renal cell carcinoma: part I-an updated review. Pharmacoeconomics. 2011;29:315-29.

5. Motzer RJ, Escudier B, McDermott DF, et al. Nivolumab versus everolimus in advanced renal-cell carcinoma. N Engl J Med. 2015;373:1803-13.

6. McDermott DF, Motzer RJ, Atkins MB, et al. Long-term overall survival (OS) with nivolumab in previously treated patients with advanced renal cell carcinoma (aRCC) from phase I and phase II studies. Presented at: 52nd Annual American Society of Clinical Oncology (ASCO) Meeting, 3-7 June 2016, Chicago, IL, USA, Abstract 4507.

7. Bohensky MA, Pasupathi K, Gorelik A, et al. A cost-effectiveness analysis of nivolumab compared with ipilimumab for the treatment of BRAF wildtype advanced melanoma in Australia. Value Health. 2016;19:1009-15.

8. Goeree R, Villeneuve J, Goeree J, et al. Economic evaluation of nivolumab for the treatment of second-line advanced squamous NSCLC in Canada: a comparison of modeling approaches to estimate and extrapolate survival outcomes. J Med Econ. 2016;19:630-44.

9. Kohn CG, Zeichner SB, Chen Q et al. Cost-effectiveness of immune checkpoint inhibition in BRAF wild-type advanced melanoma. J Clin Oncol. 2017:35:1194-202.

10. Matter-Walstra K, Schwenkglenks M, Aebi S, et al. A cost-effectiveness analysis of nivolumab versus docetaxel for advanced nonsquamous NSCLC including PD-L1 testing. J Thorac Oncol. 2016;11:1846-55.

11. Oh A, Tran DM, McDowell LC, et al. Cost-effectiveness of nivolumab-ipilimumab combination therapy compared with monotherapy for first-line treatment of metastatic melanoma in the United States. J Manag Care Spec Pharm. 2017;23:653-64.

12. Johal S, Johannesen KM, Malcolm W, Doan J. Cost-effectiveness of nivolumab in patients with advanced renal cell carcinoma in Sweden. 
Presented at: European Society for Medical Oncology (ESMO) Congress, 7-11 October 2016; Copenhagen, Denmark. Abstract 3163.

13. Wan XM, Peng LB, Ma JA, et al. Economic evaluation of nivolumab as a second-line treatment for advanced renal cell carcinoma from US and Chinese perspectives. Cancer. 2017;123:2634-41.

14. Latimer N. NICE DSU technical support document 14: survival analysis for economic evaluations alongside clinical trials —extrapolation with patient-level data. Sheffield: National Institute for Health and Clinical Excellence Decision Support Unit; 2014.

15. Wolters Kluwer Medi-Span PriceRx ${ }^{\circledR}$ database. https://pricerx.medispan. com/. Accessed July 2017

16. Perrin A, Sherman S, Pal S, et al. Lifetime cost of everolimus vs axitinib in patients with advanced renal cell carcinoma who failed prior sunitinib therapy in the US. J Med Econ. 2015;18:200-9.

17. US Department of Labor. Consumer price index. https://www.bls.gov/ cpi/detailed-report.htm. Accessed July 2017.

18. Centers for Medicare and Medicaid Services. Physician fee schedule. HCPCS code: 96413, chemo IV infusion (1 hour) - non-facility price, national payment amount. 2015.

19. Centers for Medicare and Medicaid Services. Physician fee schedule. HCPCS code: 99205, office/outpatient visit new (facility price), national payment amount. 2015

20. Motzer RJ, Porta C, Vogelzang NJ, et al. Dovitinib versus sorafenib for third-line targeted treatment of patients with metastatic renal cell carcinoma: an open-label, randomised phase 3 trial. Lancet Oncol. 2014;15:286-96.
21. Johal S, Santi I, Doan J, et al. Is RECIST-defined progression-free survival a meaningful endpoint in the era of immunotherapy? Presented at: Annual American Society of Clinical Oncology (ASCO) Genitourinary Cancers Symposium, 16-18 February 2017; Miami, FL, USA. Abstract 488.

22. Briggs AH, Claxton K, Sculpher MJ. Decision modelling for health economic evaluation. Oxford: Oxford University Press; 2006.

23. Chiou VL, Burotto M. Pseudoprogression and immune-related response in solid tumors. J Clin Oncol. 2015;33:3541-3.

24. Opdivo (nivolumab) injection [prescribing information], revised July 2017. Princeton, NJ: Bristol-Myers Squibb Company; 2017.

25. Henk HJ, Chen C, Benedict A, et al. Retrospective claims analysis of best supportive care costs and survival in a US metastatic renal cell population. Clinicoecon Outcomes Res. 2013;5:347-54.

26. Hollenbeak CS, Nikkel LE, Schaefer EW, et al. Determinants of medicare all-cause costs among elderly patients with renal cell carcinoma. J Manag Care Pharm. 2011;17:610-20.

27. Kim SP, Gross C, Abouassaly R, et al. Out-of-pockets costs for patients receiving targeted agents for metastatic renal cell carcinoma. J Clin Oncol. 2014;32. Abstract e15598.

28. Cella D, Grunwald V, Nathan P, et al. Quality of life in patients with advanced renal cell carcinoma given nivolumab versus everolimus in CheckMate 025: a randomised, open-label, phase 3 trial. Lancet Oncol. 2016;17:994-1003.

\section{Submit your next manuscript to BioMed Central and we will help you at every step:}

- We accept pre-submission inquiries

- Our selector tool helps you to find the most relevant journal

- We provide round the clock customer support

- Convenient online submission

- Thorough peer review

- Inclusion in PubMed and all major indexing services

- Maximum visibility for your research

Submit your manuscript at www.biomedcentral.com/submit
() Biomed Central 